\title{
The relationship between nasal index and nasal airway resistance, and response to a topical decongestant*
}

\author{
N.M. Doddi and R. Eccles \\ Common Cold Centre and Healthcare Clinical Trials, Cardiff School of Biosciences, \\ Cardiff University, Cardiff, United Kingdom
}

\begin{abstract}
SUMMARY The differences in the shape and size of the nose have been proposed to be an adaptation to climate with broad noses (platyrrhine) evolving in a warm humid environment where there was little need for air conditioning and narrow noses (leptorrhine) evolving in colder climates where the air needed more warming. The main aim of this research was to determine if there was any relationship between the shape of the nose as expressed in terms of nasal height and width (nasal index) and total nasal airway resistance (NAR), as one would predict that the narrower leptorrhine noses would have a greater resistance to air flow than the broader platyrrhine noses. It was also proposed that the narrow leptorrhine nose would have better developed vascular tissue than the broad platyrrhine nose in order to condition cold air, and would exhibit a greater response to nasal decongestion. No correlation was found between nasal index and NAR $(r=-0.09)$ and similarly no correlation was found between nasal index and response to a topical nasal decongestant $(r=0.02)$. The absence of any physiological differences between the different nose types may be due to acclimatisation of participants to the area of recruitment.
\end{abstract}

Key words: nasal index, rhinomanometry, nasal airway resistance, nasal decongestant

\section{INTRODUCTION}

The shape and size of the human nose are often described as leptorrhine, mesorrhine and platyrrhine, and these classifications correspond to differences in nasal height and width expressed as a nasal index ${ }^{(1)}$. The three forms of nose are classified according to the calculated nasal index $<70,70-$ 85 and $>85$ respectively ${ }^{(1)}$. The differences in the shape and size of the nose have been proposed to be an adaptation to climate with the large open platyrrhine nose having evolved in a warm humid environment where there was little need for air conditioning and the narrow leptorrhine nose having evolved in colder climates where the air needed more warming ${ }^{(2,3)}$. Although there has been research on the variation in the nasal index with climate there has been little research to determine if there are any physiological differences associated with variation of the nasal index. The aim of this research was to determine if there is any relationship between nasal index and total nasal airway resistance (NAR), as one would predict that the narrower leptorrhine noses would have a greater resistance than the broader platyrrhine noses, with the mesorrhine noses having an intermediate position. The higher resistance of the leptorrhine nose has been reported to be due to adaptation to a cold climate where greater warming of the inspired air is needed compared to the platyrrhine nose which is adapted to a warm humid climate where there is little need to condition the inspired air ${ }^{(2)}$.

Previous research on the relationship between race and NAR can be criticised by the way the participants were recruited and categorised as Caucasian, Oriental or Negro ${ }^{(4,5)}$ rather than basing recruitment on more objective measurements of the nose such as the nasal index. This centre has recently proposed that the nasal index should be used to categorise subjects in rhinology research and that the use of racial categories such as Caucasian, Oriental etc. should be discouraged as unscientific ${ }^{(6-8)}$.

A secondary aim of the research was to determine if there was any difference in the response of the nasal airway to application of a topical nasal decongestant. The air conditioning capacity of the nose has been proposed to be related to the thickness of the nasal mucosa and the development of the large veins that warm the inspired air ${ }^{(2)}$. If the nasal venous sinusoids are better developed in the narrow noses adapted to a cold climate (leptorrhine) compared to the broad noses adapted to a warm tropical climate, then one would expect a greater decongestant action of a topical decongestant (i.e. greater change in airflow) in the leptorrhine nose compared to the platyrrhine nose. 


\section{MATERIALS AND METHODS}

\section{Ethical approval}

The study was reviewed and approved by the South East Wales Local Research Ethics Committee and conducted in accordance with the International Conference of Harmonization's 'Guidelines for Good Clinical Practice' and the World Medical Association's 'Declaration of Helsinki'.

\section{Subjects}

Subjects that responded to an advertisement by poster or email, were screened by the investigator to ensure that they met the inclusion and exclusion criteria for the study. Anterior rhinoscopy using a Thuddicum speculum was performed to detect any gross nasal septal deviation or the presence of nasal polyps. In order to qualify for entry to the study patients had to be aged 18 years and above and give written informed consent. Subjects were excluded from the study if they were suffering from any nasal disease (nasal polyps, chronic rhinitis, severe septal deviation) or other clinically significant disease, if they had a history of any nasal surgery, ingested any alcohol in the previous 12 hours or more than 4 units of alcohol in the previous 24 hours, were a drug or alcohol abuser, were taking any medication that could influence the nose or that was contraindicated for use with a topical nasal decongestant, had ingested any hot food or drink within the previous hour, had taken any confectionery or other product containing menthol in the previous hour, were suffering from a common cold/flu like illness or had a history of cold or flu within the previous two weeks, had current nasal allergic disease or were taking any medicine to treat nasal allergic disease, had taken any nasal decongestant medication or nasal spray treatment in the previous 24 hours, had a history of trans-sphenoidal hypophysectomy, had known hypersensitivity to or any idiosyncratic reaction to xylometazoline or excipients of nasal sprays, used any antidepressant drugs or monoamine oxidase inhibitors, had a diagnosis of arterial hypertension that was not well controlled.

\section{Procedures}

The study was conducted as follows; measurement of nose to determine nasal index, allocation to nasal index cohort, subjective scoring of nasal airflow, rhinomanometry to measure NAR, administration of topical nasal decongestant, subjective scoring of nasal airflow 30 minutes after decongestion, rhinomanometry to measure NAR.

\section{Measurement of nose}

Subjects had the height and width of the nose measured to determine the nasal index. The nasal index in this study was measured as described by Oladipo (2009) ${ }^{(9)}$. The height of the nose was measured with a sliding calliper, from nasion to nasospinale. The nasal breadth (maximum breadth of the nose) was measured at a right angle to the nasal height from ala to ala. The nasal index was calculated as follows (Width of the nose $\mathrm{x} 100 /$ height of the nose) ${ }^{(2)}$. Subjects were allocated to one of six cohorts for nasal index as follows $-<60,60-<$ $65,65-<70,70-<75,75-<80,>80$.
Each cohort recruited up to a maximum of 10 subjects. The range of nasal index is based on a recent literature review of the nasal index ${ }^{(7)}$ and the work of earlier researchers ${ }^{(1)}$. Illustrations of the range of nasal shapes according to nasal index and the geographical distribution of nasal index can be found in a recent review article on this topic ${ }^{(7)}$.

\section{Rhinomanometry}

An NR6-2 rhinomanometer (GM instruments, Glasgow, UK) was used in this study to measure NAR using the technique of posterior rhinomanometry. The instrument measured total nasal airflow at a sample pressure of $75 \mathrm{~Pa}$. Subjects breathed into a facemask whilst sealing their lips around a pressure sensing tube in the oral cavity. For each measurement of NAR two consecutive sets of four respiratory cycles were obtained and the coefficient of variation (CV) of these two sets was calculated. If the $\mathrm{CV}$ was $10 \%$ or less, the measurement was accepted and the mean NAR of the 8 respiratory cycles was calculated. If the $\mathrm{CV}$ was more than $10 \%$ then the respiratory cycle sets were discarded and the procedure repeated until a $\mathrm{CV}$ of $10 \%$ or less was achieved. If a CV less than $10 \%$ could not be obtained after three attempts the data was entered as missing. The results for rhinomanometry were expressed as both NAR and airflow conductance (sample pressure $75 \mathrm{~Pa}$ divided by resistance $=$ airflow conductance) .

\section{Subjective symptom scores}

A $100 \mathrm{~mm}$ visual analogue scale (VAS) anchored by the descriptors 'nose feels extremely clear $(0 \mathrm{~mm})$ and 'nose feels extremely blocked' (100 mm) was used to assess the sensation of nasal airflow prior to measurement of NAR. The scores represented the sensation of nasal airflow at the time of measurement.

\section{Nasal decongestant spray}

The nose was decongested by Otrivine Adult Nasal spray. The decongestant nasal spray is manufactured by Novartis Consumer Health and contains the sympathomimetic vasconstrictor medicine xylometazoline $0.1 \% \mathrm{w} / \mathrm{v}$. The investigator administered the normal dose of 1 spray in each nostril, and the subject was asked to sniff the spray gently into the nose rather than allowing it to run out of the nose.

\section{Sample size, statistics and efficacy variables}

This was a pilot study and the magnitude of any relationship between nasal index and nasal airway resistance was unknown as there are no comparable studies in the literature. A study number of 10 healthy subjects for each of the six cohorts of nasal index was chosen to provide sufficient data for exploratory analysis.

The primary objective of the study was to determine if there was a correlation between nasal index and NAR. Secondary objectives were to determine if there was any relationship between nasal index and changes in NAR and subjective scores of nasal airflow after decongestion of nasal blood vessels. A null hypothesis for this study was that there is no relationship between nasal index and NAR of the decongested nose. 


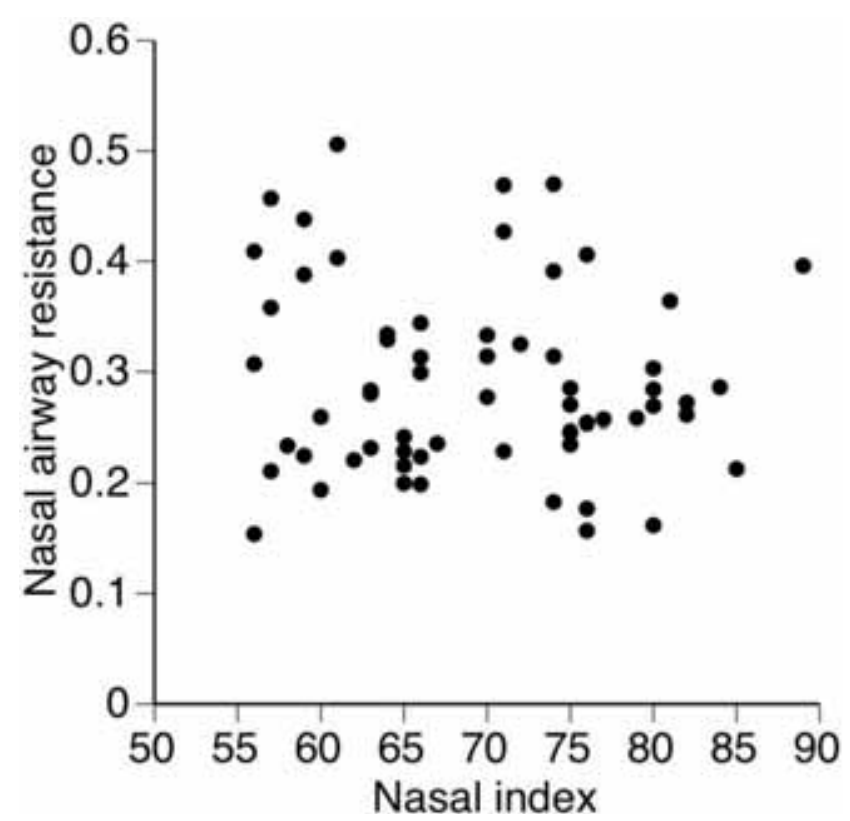

Figure 1. Relationship between nasal airway resistance $\left(\mathrm{Pa} \mathrm{cm}{ }^{3} \mathrm{sec}\right)$ and nasal index.

The Statistical Package for the Social Sciences version 11 (SPSS 11) and the Microsoft Excel 2008 version 12.1.9 for the Macintosh platform was utilised for statistical analysis. It was assumed that the NAR data was not normally distributed and all data was analysed with the non-parametric Wilcoxon signed ranks test. Results for nasal conductance have a normal distribution and are presented as means with standard deviation (S.D.).

\section{RESULTS}

\section{Subjects}

A total of 69 healthy participants were screened to recruit the 60 participants. Eight of 69 participants screened were unable to perform the posterior rhinomanometry and were excluded. The 61 participants were grouped into 6 groups based on their nasal index. The groups were as follows: nasal index of $<60$, $60-64,65-69,70-74,75-79$ and $>80$. There were 10 subjects in each group except the group with nasal index $75-79$, which had 11 subjects.

There were 31 males and 30 females. The mean age was 23.05 years with a range from 18 to 46 years. There were 33 Caucasians, 23 Asians and 5 participants of Negroid race. The race was self-determined by the participants, and for those of mixed race the race of the father was used to determine racial classification. The minimum height was $150 \mathrm{~cm}$ and the maximum was $191 \mathrm{~cm}$ with an average of $170.21 \mathrm{~cm}$. The lowest weight among the participants was $38 \mathrm{~kg}$ while highest weight was $98 \mathrm{~kg}$ with an average of $65.46 \mathrm{~kg}$. There were no differences in body mass index (BMI) between the groups.

The past medical history included 1 participant with mild dust allergy and one participant with hay fever but was out of season for his allergy. Both participants had no recent history of allergic reaction.

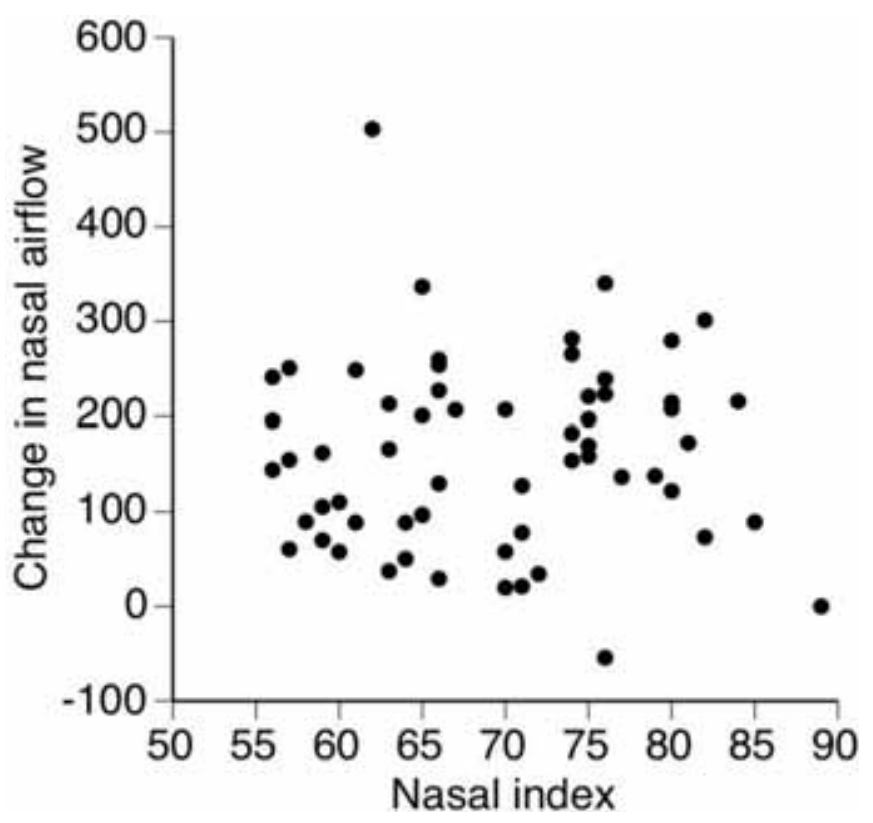

Figure 2. Relationship between change in nasal airflow on application of a topical decongestant (expressed as change in conductance, $\mathrm{cm}^{3}$ sec) and nasal index.

Nasal index and nasal airflow

The correlation between total NAR and nasal index is illustrated in Figure 1. The Figure demonstrates that there is no clear relationship between NAR and nasal index $(r=-0.09)$. The results for the decongested nose after application of the topical nasal decongestant also show no relationship between NAR and nasal index $(r=-0.02)$. Similarly there was no relationship between the subjective sensation of nasal airflow as obtained by VAS sores and NAR both before $(r=0.04)$ and after decongestion $(r=0.04)$.

Degree of decongestion and nasal index

The correlation between the change in nasal airflow (change in nasal conductance) after decongestion and the nasal index is illustrated in Figure 2. The Figure demonstrates that there is no correlation between the change in nasal airflow (i.e. the degree of decongestion) and the nasal index $(\mathrm{r}=0.02)$. The mean nasal conductance before decongestion was $278.72 \mathrm{~cm}^{3} \mathrm{sec}$ (SD: 80.49, $\mathrm{n}=61)$ and this increased to $438.5 \mathrm{~cm}^{3} \mathrm{sec}(\mathrm{SD}: 129.94, \mathrm{n}=61)$ after application of the topical decongestant.

In total, 59/61 participants responded to the application of the topical decongestant with an increase in nasal airflow ranging from $29 \mathrm{~cm}^{3} \mathrm{sec}$ to $503 \mathrm{~cm}^{3} \mathrm{sec}$ (mean change in conductance $\left.159.78 \mathrm{~cm}^{3} \mathrm{sec}, \mathrm{SD}: 98.68, \mathrm{n}=61\right)$. One participant had no change in nasal airflow. One participant responded to the decongestant with a paradoxical decrease in nasal airflow (-54 $\left.\mathrm{cm}^{3} \mathrm{sec}\right)$. This participant was recalled and the decongestant procedure was repeated and the participant still responded with a decrease in nasal airflow $\left(-50 \mathrm{~cm}^{3} \mathrm{sec}\right)$. 


\section{DISCUSSION}

The shape of the human nose has been shown to vary with climate with the narrow leptorrhine nose found in cold dry climates and the broad plattyrrhine nose in hot humid climates. The shape and size of the nose can be defined in terms of the nasal index, which takes into consideration both the height and width of the nose, and the nasal index has been reported to correlate with climatic conditions ${ }^{(1,2,10)}$. The nasal index correlates with both air temperature $(r=0.63)$ and humidity $(r=0.42)$ and with a combination of both temperature and humidity $(\mathrm{r}=0.72)^{(2)}$. The aim of the present study was to determine if there was any correlation between nasal index and NAR, as the narrow leptorrhine nose with a low nasal index found in cold dry climates, may be expected to have a higher NAR than the broad platyrrhine nose found in hot humid climates. The cosmopolitan population of a British university allowed us to recruit participants with a wide range of nasal index measures.

The results of the study did not find any evidence for any relationship between nasal index and nasal airway resistance in both the normal and decongested nose. This lack of correlation may be due to the small sample size of the study but it may also represent the true condition of the larger population, as Figure 1 does not show any trend for correlation of the nasal index and NAR. Although the participants had a wide range of nasal index they were all recruited in the same area of Wales in the United Kingdom, and therefore were all acclimatised to the Welsh temperate climate. It is possible that over a period of time the nose of an individual adapts to climate and that this adaptation eliminates any differences in NAR between leptorrhine and platyrrhine noses. The nose does respond to acute changes in air temperature with changes in NAR ${ }^{(11,12)}$. Acute cold air exposure causes an increase in NAR ${ }^{(12)}$, and this acute response could develop into a longer-term increase in NAR. Platyrrhine noses with a normally low NAR in a hot humid climate could acclimatise to the colder Welsh climate with an increase in NAR.

Objective and subjective measures of total nasal airflow do not correlate, and therefore a subjective measure of nasal airflow was used in this study to complement the objective rhinomanometry. However, no relationship was found between nasal index and subjective scores of nasal airflow.

The overall response to the application of the topical decongestant was an increase in nasal conductance and there was no correlation between the nasal index and the change in conductance as illustrated in Figure 2. If there was any difference in the development of the nasal venous sinusoids in the different nose types one would have expected a greater decongestant response in the leptorrhine nose compared to the plattyrrhine nose. Again, the lack of any correlation between nasal index and nasal decongestant response could be due to acclimatisation of participants to the same Welsh climatic conditions. The results demonstrate that a wide range of different nasal types all give similar decongestant responses to the nasal decongestant spray. The paradoxical decrease in conductance in response to the topical decongestant in one participant cannot at present be explained and a survey of the literature has not found any similar case reports.

\section{CONCLUSIONS}

The results demonstrate that no correlation was found between the nasal index and NAR, and also between the nasal index response to a topical nasal decongestant. The absence of any physiological differences between the different nose types may be due to acclimatisation of participants to the area of recruitment.

\section{ACKNOWLEDGEMENT}

The study was financed from the research funds of the Common Cold Centre, Cardiff University and also received a small unencumbered donation and gift of nasal decongestant sprays from Novartis Consumer Healthcare.

\section{REFERENCES}

1. Davies A. A re-survey of the morphology of the nose in relation to climate. J Roy Anthrop Inst. 1932; 62: 337-359.

2. Thomson A, Dudley Buxton L. Man's nasal index in relation to certain climatic conditions. J Roy Anthrop Inst. 1923; 53: 99-122.

3. Wolpoff MH. Climatic influence on the skeletal nasal aperture. Am J Phys Anthropol. 1968; 29: 405-423.

4. Ohki M, Naito K, Cole P. Dimensions and resistances of the human nose: racial differences. Laryngoscope. 1991; 101: 276-278.

5. Canbay EI, Bhatia SN. A comparison of nasal resistance in white Caucasians and blacks. Am J Rhinol. 1997; 11: 73-75.

6. Leong SC, Eccles R. What defines a Thai, Caucasian, or Asian? Am J Rhinol Allergy. 2009; 23: 238.

7. Leong SC, Eccles R. A systematic review of the nasal index and the significance of the shape and size of the nose in rhinology. Clin Otolaryngol. 2009; 34: 191-198.

8. Leong SC, Eccles R. The use of race as a demographic variable in clinical research. Rhinology. 2010; 48: 3-6.

9. Oladipo G, Eroje M, Fahwehinmi H. Anthropometric comparison of nasal indices between Andoni and Okrika tribes of Rivers State Nigeria. International Journal of Medicine and Medical Sciences. 2009; 1: 135-137.

10. Davies A. Mans nasal index in relation to climate. Man. 1929; 29: 8-14.

11. Proctor DF, Andersen I, Lundqvist GR. Human nasal mucosal function at controlled temperatures. Respiration physiology. 1977; 30: 109-124.

12. Cole P, Forsyth R, Haight JSJ. Effects of cold air and exercise on nasal patency. Annals of Otology, Rhinology and Laryngology. 1983; 92: 196-198.

\author{
Professor Ron Eccles \\ Common Cold Centre and Healthcare Clinical Trials \\ Cardiff School of Biosciences \\ Cardiff University \\ Cardiff CF10 3 US \\ United Kingdom
}

Tel: +44-(0)292-087 4102

Fax: +44-(0)292-087 4093

E-mail: eccles@cardiff.ac.uk 